8. Frobenius, "Ueber die Leibnitzsche Reihe," Journal für Mathematik, volume 89 (1880).

9. Gronwall, "Ueber die Laplacesche Reihe," Mathematische Annalen, volume 74 (1913)

10. Gronwall, "Ueber die Summierbarkeit der Reihen von Laplace und Legendre," Mathematische Annalen, volume 75 (1914).

11. Haar, "Zur Theorie der orthogonalen Funktionensysteme," Dissertation, Göttingen (1909), and Mathematische Annalen, volume 69 (1910).

12. Haar, "Ueber die Legendresche Reihe," Rendiconti del Circolo Matematico di Palermo, volume 32 (1911).

13. G. H. Hardy, "On the summability of Fourier's series," Proceedings of the London Mathematical Society, series 2, volume 12 (1913).

14. Lebesgue, "Recherches sur la convergence des séries de Fourier," Mathematische Annalen, volume 61 (1905).

15. C. N. Moore, "The summability of the developments in Bessel functions, with applications," Transactions of the American Mathematical Society, volume 10 (1909).

16. C. N. Moore, "On convergence factors in double series and the double Fourier's series," Transactions of the American Mathematical Society, volume 14 (1913).

17. C. N. Moore, "On the summability of the double Fourier's series of discontinuous functions," Mathematische Annalen, volume 74 (1913).

18. Marcel Riesz, "Sur les séries de Dirichlet et les séries entières," Comptes Rendus, volume 149 (1909).

19. W. H. Young, "Ueber eine Summationsmethode für die Fouriersche Reihe," Leipziger Berichte, volume 53 (1911).

20. W. H. Young, "On infinite integrals involving a generalization of the sine and cosine functions," Quarterly Journal of Mathematics, volume $43(1912)$

21. W. H. Young, "On multiple Fourier series," Proceedings of the London Mathematical Society, series 2, volume 11 (1912).

\title{
MODULAR SYSTEMS.
}

The Algebraic Theory of Modular Systems. By F. S. MacauLAY. [Cambridge Tracts in Mathematics and Mathematical Physics, No. 19.] Cambridge University Press, 1916. xiv $+112 \mathrm{pp}$.

A MODULAR system is an infinite aggregate of polynomials in $n$ variables $x_{1}, x_{2}, \cdots, x_{n}$, defined by the property that if $F, F_{1}, F_{2}$ belong to the system, $F_{1}+F_{2}$ and $A F$ also belong to the system, where $A$ is any polynomial in $x_{1}, x_{2}, \cdots, x_{n}$. Hence if $F_{1}, F_{2}, \cdots, F_{k}$ belong to a modular system so also does $A_{1} F_{1}+A_{2} F_{2}+\cdots+A_{k} F_{k}$, where $A_{1}, A_{2}, \cdots, A_{k}$ are arbitrary polynomials in $x_{1}, x_{2}, \cdots, x_{n}$. In the algebraic theory (to which this tract is devoted) polynomials such as $F$ and $a F$, where $a$ is a quantity not involving the variables, are regarded as the same polynomial. 
Let us consider any given infinite aggregate of polynomials in $x_{1}, x_{2}, \cdots, x_{n}$. Of those of any given degree $L$ there are only a finite number which are linearly independent. Hence we may select an ordered sequence of polynomials $F_{1}, F_{2}, F_{3}, \ldots$ of the given aggregate such that any given polynomial of the aggregate is linearly expressible in terms of a finite number of the polynomials $F_{1}, F_{2}, F_{3}, \cdots$. Concerning such an ordered enumerable sequence of polynomials Hilbert has established the following theorem:

If $F_{1}, F_{2}, F_{3}, \cdots$ is an infinite sequence of polynomials in $n$ variables $x_{1}, x_{2}, \cdots, x_{n}$, then there exists a finite number $k$ such that for $h>k$ we have a relation of the form

$$
F_{h}=A_{h 1} F_{1}+A_{h 2} F_{2}+\cdots+A_{h k} F_{k}
$$

in which the $A_{i j}$ denote polynomials in $x_{1}, x_{2}, \cdots, x_{n}$.

Our author gives on page 38 essentially König's proof of this theorem. Since the proof is from first principles, he is justified in assuming the theorem from the beginning.

From the foregoing considerations it follows that every infinite aggregate of polynomials is contained in the infinite aggregate of a modular system and that every modular system is itself the aggregate of polynomials

$$
A_{1} F_{1}+A_{2} F_{2}+\cdots+A_{k} F_{k}
$$

in which $F_{1}, F_{2}, \cdots, F_{k}$ are $k$ properly chosen polynomials of the system and $A_{1}, A_{2}, \cdots, A_{k}$ are arbitrary polynomials. The polynomials $F_{1}, F_{2}, \cdots, F_{k}$ are said to form a basis of the system.

In its simpler aspects the theory of modular systems is of importance in geometry. Let it be required, for example, to determine the class of algebraic plane curves which pass through the intersection points of the curves

$$
F_{1}(x, y)=0, \quad F_{2}(x, y)=0 .
$$

It is obvious that the class includes all the curves

$$
A_{1} F_{1}+A_{2} F_{2}=0
$$

where $A_{1}$ and $A_{2}$ are polynomials in $x$ and $y$. The question arises as to whether or under what circumstances every algebraic curve passing through the given intersection points can be represented by an equation of the last form above. The 
answer to such a question affords one of the simplest applications of the algebraic theory of modular systems.

The primary object of the algebraic theory of modular systems is to discover those general properties of a system which will afford a means of answering the question whether a given polynomial is or is not a member of a given system. This calls for a generalization of the theory of the solution of equations in several unknowns. In order that a polynomial $F$ may belong to a modular system with a given basis $F_{1}, F_{2}$, $\cdots, F_{k}$ it is obviously necessary that $F$ shall vanish for all finite solutions of the system $F_{1}=F_{2}=\cdots=F_{k}=0$. This condition is sufficient only if the given modular system has a certain property; otherwise, it is not sufficient, and $F$ must satisfy further conditions, also connected with the solutions of the system $F_{1}=F_{2}=\cdots=F_{k}=0$; and these may be difficult to express concretely.

At present the theory of modular systems is incomplete and offers a wide field for research. The subject is one of great difficulty, and he who works in it must exercise extreme care to avoid the pitfalls. Macaulay points out several mistakes made by his predecessors, some of them occurring in the most important memoirs dealing with the subject.

From what has been said it is evident that the first step in the theory of modular systems is to find all the solutions of the algebraic equations $F_{1}=F_{2}=\cdots=F_{k}=0$. This is completely accomplished in the theories of the resultant and the resolvent, developed by our author in sections I and II (pages 3-28). The theory of the resultant of two homogeneous polynomials in two variables is first treated. There is given then a parallel development of the much more difficult theory of the resultant of $n$ homogeneous polynomials in $n$ variables. From this the necessary theory for non-homogeneous polynomials follows at once. Application is made to the solution of certain classes of algebraic equations. In the theory of the resolvent the author follows in the main König's exposition of Kronecker's method of solving equations by means of the resolvent.

General properties of modular systems are developed in section III (pages 29-63) The treatment is closely allied to Lasker's memoir (Mathematische Annalen, volume 60, 1905) and Dedekind's theory of ideals. Section IV (pages 64-100) contains an extension of Lasker's results founded on the methods 
originated by Noether. The contents of this chapter are due to the author himself. A considerable number of the properties proved in the section have been established by him in previous memoirs. But a new method, that of the inverse system, is here employed for the first time and the results are closely associated with it. The author's own account of the method is to be found on pages $64 \mathrm{ff}$.

The monograph ends with a note of twelve closely printed pages containing a brief explanation of the theory of ideals of algebraic numbers and functions and of the relation in which the algebraic theory of modular systems stands with respect to it.

Throughout the tract the exposition is given in condensed form, evidently best adapted to the needs of investigators in the field. But a portion of the treatment, especially that of the first two sections, is suited to the needs of the general mathematical reader interested in the general aspects of the theory of algebraic equations in several unknown quantities. R. D. Carmichael.

\section{NOTES.}

THE regular meeting of the Chicago Section of the American Mathematical Society at the University of Chicago on Friday and Saturday, April 4-5, 1919, will include a symposium on the geometry of numbers with applications to questions of minima and algebraic numbers. Formal papers, based largely on the work of Minkowski, will be presented by Professor $\mathrm{H}$. F. Blichfeldt, of Stanford University, and Professor L. E. Dickson, of the University of Chicago. Synopses of these papers will be sent out with the programmes of the meeting.

THE programme of the regular meeting of the Society in New York City on April 26, 1919, will include reports of the work of members of the Society in the government Ordnance Department at Washington and Aberdeen.

The opening (January) number of volume 20 of the Transactions of the American Mathematical Society contains the following papers: "Necessary conditions in the problems of 